\title{
JA signal-mediated immunity of Dendrobium catenatum to necrotrophic Southern Blight pathogen
}

\author{
Cong $\mathrm{Li}^{1 \dagger}$, Qiuyi Shen ${ }^{1 \dagger}$, Xiang Cai ${ }^{1}$, Danni Lai ${ }^{1}$, Lingshang Wu' ${ }^{1}$ Zhigang Han ${ }^{1}$, Tianlun Zhao², \\ Donghong Chen ${ }^{1 *}$ and Jinping $\mathrm{Si}^{1^{*}}$
}

\begin{abstract}
Background: Dendrobium catenatum belongs to the Orchidaceae, and is a precious Chinese herbal medicine. In the past 20 years, D. catenatum industry has developed from an endangered medicinal plant to multi-billion dollar grade industry. The necrotrophic pathogen Sclerotium delphinii has a devastating effection on over 500 plant species, especially resulting in widespread infection and severe yield loss in the process of large-scale cultivation of $D$. catenatum. It has been widely reported that Jasmonate (JA) is involved in plant immunity to pathogens, but the mechanisms of JA-induced plant resistance to $S$. delphinii are unclear.
\end{abstract}

Results: In the present study, the role of JA in enhancing D. catenatum resistance to $S$. delphinii was investigated. We identified 2 COI1, $13 \mathrm{JAZ}$, and 12 MYC proteins in D. catenatum genome. Subsequently, systematic analyses containing phylogenetic relationship, gene structure, protein domain, and motif architecture of core JA pathway proteins were conducted in D. catenatum and the newly characterized homologs from its closely related orchid species Phalaenopsis equestris and Apostasia shenzhenica, along with the well-investigated homologs from Arabidopsis thaliana and Oryza sativa. Public RNA-seq data were investigated to analyze the expression patterns of $D$. catenatum core JA pathway genes in various tissues and organs. Transcriptome analysis of MeJA and S. delphinii treatment showed exogenous MeJA changed most of the expression of the above genes, and several key members, including DcJAZ1/2/5 and DCMYC2b, are involved in enhancing defense ability to S. delphinii in D. catenatum.

Conclusions: The findings indicate exogenous MeJA treatment affects the expression level of $D c J A Z 1 / 2 / 5$ and DCMYC2b, thereby enhancing D. catenatum resistance to $S$. delphinii. This research would be helpful for future functional identification of core JA pathway genes involved in breeding for disease resistance in D. catenatum.

Keywords: Dendrobium catenatum, Jasmonate, Sclerotium delphinii, COI1, JAZ, MYC transcription factor

\section{Background}

Dendrobium of Orchidaceae is an importantly economical genera, which comprises nearly 1,600 accepted species $[1,2]$. Among them, D. catenatum (also known as

\footnotetext{
*Correspondence: donghong.chen@zafu.edu.cn; Issjp@163.com

${ }^{\dagger}$ Cong Li and Qiuyi Shen contributed equally to this work.

1 State Key Laboratory of Subtropical Silviculture, Zhejiang A\&F University, Hangzhou 311300, China

Full list of author information is available at the end of the article
}

D. officinale) is a popular tonic and traditional Chinese medicine herb with prominent commercial value, including protection against upset stomach, promotion of body fluid production, immune enhancement, nourishing Yin, as well as antioxidant and antitumor effects [3]. In the past 20 years, scientists have successfully overcome the bottleneck problems against $D$. catenatum industrious development, such as seedling breeding and facility cultivation [3], which makes it from an endangered medicinal plant to multi-billion dollar grade industry [4]. However, 
in the process of large-scale cultivation of $D$. catenatum, Southern Blight disease caused by Sclerotium delphinii leads to heavy yield losses by infecting its leaves, stems, and roots [5].

Southern Blight is a broad-spectrum disease caused by the necrotrophic pathogen $S$. delphinii and S. rolfsii $[5,6]$. Athelia rolfsii is the sexual state of the fungus, we rarely see it, while its asexual state $S$. delphinii and S. rolfsii are more common [6]. Compared with S. rolfsii, S. delphinii is more pathogenic [5]. In warm and high humidity environments, $S$. delphinii infects the host and secretes many cell wall-degrading enzymes (CWDEs) to degrade the host cell wall [7], and then hyphae penetrate the plant tissue causing local necrosis, with subsequent formation of abundance of white mycelium and rapeseed-like sclerotia [5]. Southern Blight is considered one of the most destructive diseases worldwide for its vast host range of over 500 plant species, including various crops, vegetables, fruits, ornamental flowers, medicinal plants, etc., resulting in an average economic loss of $25 \%$ [8], and even $80 \%$ in severe cases [7]. Furthermore, its sclerotia can survive in soil for over five years even in the absence of hosts [7]. At present, biological control agents including Rhizobium [9], Trichoderma [10], and Streptomyces [7], and chemical control fungicides like tebuconazole and flutolanil [11], have been reported. However, biocontrol has the problem of inefficiency and fungicide utilization leads to bio-magnification in the food chain and environmental pollution [9]. Therefore, new control strategy is urgently needed.

The lipid-derived hormones, jasmonates (JAs), regulate plants defense against necrotrophic pathogens and a wide variety of herbivores [12-14]. Necrotrophic pathogens affected by JA-induced plant defenses include the bacterial pathogens Xanthomonas oryzae and Pectobacterium atrosepticum [15], fungal pathogens such as Alternaria brassicicola, Botrytis cinerea, Sclerotinia sclerotiorum, Plectosphaerella cucumerina, Fusarium oxysporum, and oomycete Pythium spp [12,13]. The bioactive form jasmonoyl-L-isoleucine (JA-Ile) is produced by conjugation of jasmonic acid with isoleucine in the plant cytosol [16], which is perceived through a nuclear co-receptor composing of CORONATINE INSENSITIVE1 (COI1), the F-box subunit of the ubiquitin ligase SCF $^{\mathrm{COI}}$ [17], and JASMONATE ZIM-DOMAIN (JAZ) proteins [18-21]. In Arabidopsis, the COI1 gene encodes a $66-\mathrm{kD}$ protein with 16 leu-rich repeats (LRRs) and an F-box motif [22]. In 'stress-free' conditions with low levels of JA-Ile, JA responses are repressed by a group of JAZ proteins [16, 19-21]. JAZs belong to the TIFY superfamily that is defined by a highly conserved TIFY motif (TIF[F/Y] XG) residing within the ZIM domain [19-21, 23]. In Arabidopsis, 13 genes (JAZ1-JAZ13) encode JAZ proteins with a highly conserved Jas motif (also known as a CCT_2 motif) at the C terminus [24, 25]. JAZ proteins have a strong affinity on some related bHLH TFs such as MYC2, MYC3, MYC4, and MYC5 as well as GL3 (GLA BRA3), EGL1 (ENHANCE R OF GLA BRA3 1), and TT8 (TRANSPARENT TE STA 8), involved in both specific and overlapped pathways of JA [26, 27]. These bHLHtype MYC TFs share highly sequence homology both in bHLH and bHLH-MYC_N (containing JAZ-interacting domain (JID) domain) domains [27, 28]. Of these JAZ targeted TFs, MYC2 is best-characterized and considered as a regulatory hub of the JA signaling pathway [29-31]. JAZ repressors physically bind and inhibit MYCs [20, 24] through two distinct mechanisms [19-21]. First, MYCbound JAZ proteins recruit the co-repressors TOPLESS (TPL) either directly by ETHYLENE-RESPONSE FACTOR Amphiphilic Repression (EAR) motifs located at the $\mathrm{N}$ terminus of a subset of JAZ proteins (e.g., JAZ7, JAZ8, JAZ13) or indirectly through NOVEL INTERACTOR OF JAZ (NINJA), an EAR motif-containing protein [32-34]. Second, the Jas motif of JAZ binding to the N-terminal JID of MYCs blocks access of MYCs to the MED25 coactivator subunit of the mediator complex [35-37]. Several JAZ repressors belong to AtJAZI and AtJAZIII clade also contain an N-terminal cryptic MYC-interaction domain (CMID), which binds MYCs more tightly than the Jas motif [26, 38-42]. In response to stress cues, JA-Ile was synthesized, which directly promotes the interaction between COI1 and JAZ, and subsequent $\mathrm{SCF}^{\mathrm{CO} I 1}$-dependent degradation of JAZ repressors occurred $[18,43]$. The liberation of MYCs from repressive state results in extensive transcriptional reprograming and a plethora of JA-dependent physiological responses $[44,45]$. Hence, it is significant to exploit the role of JA pathway genes against Southern Blight in $D$. catenatum molecular breeding.

Here, we employed a genetic approach to identify the members of COI, JAZ, and MYC family of $D$. catenatum, respectively, and subsequently assessed the phylogenetic relationship, gene structure, and domain architecture, and then showed exogenous MeJA enhanced D. catenatum resistance to $S$. delphinii through phenotype identification and gene expression profiling in the core JA pathway. The results provide the basis for further functional characterization of DcCOIs, DcJAZs, and DcMYCs, and in particular, their role in $D$. catenatum response to S. delphinii.

\section{Results}

Exogenous jasmonates enhanced D. catenatum resistance to S. delphinii

Pre-treated with $1 \mathrm{mM}$ MeJA for $4 \mathrm{~h}$, four-month-old D. catenatum plantlets were inoculated with $S$. delphinii 
mycelia suspensions. Different responses to the disease infection were observed in control and MeJA-pretreated plantlets (Fig. 1). The symptoms, water-stained and brown necrotic lesions, were much more severe in the control than in pre-treated plantlets at 60 hpi (Fig. 1A). With prolonged hpi, the disease became more and more serious, but disease development was much slower in the pre-treated plantlets compared with the control. At 144 hpi (6 dpi), the disease index was 82.50 and $33.33 \%$ for the control and MeJA pre-treated plantlets, respectively (Fig. 1B). While almost all control plantlets were nearly dead and covered with white sclerotia at $240 \mathrm{hpi}$ (10 dpi), pre-treated plantlets showed less necrotic lesions and no visible mycelia or sclerotia (Fig. 1A).

\section{Identification of D. catenatum core JA signaling pathway genes}

In view of the above-stated phenotypic results, we screened and identified the JA pathway genes in D. catenatum. The COI protein sequences of Arabidopsis and rice were used as queries to BLASTP search against the $D$. catenatum and its close relative $P$. equestris and $A$.

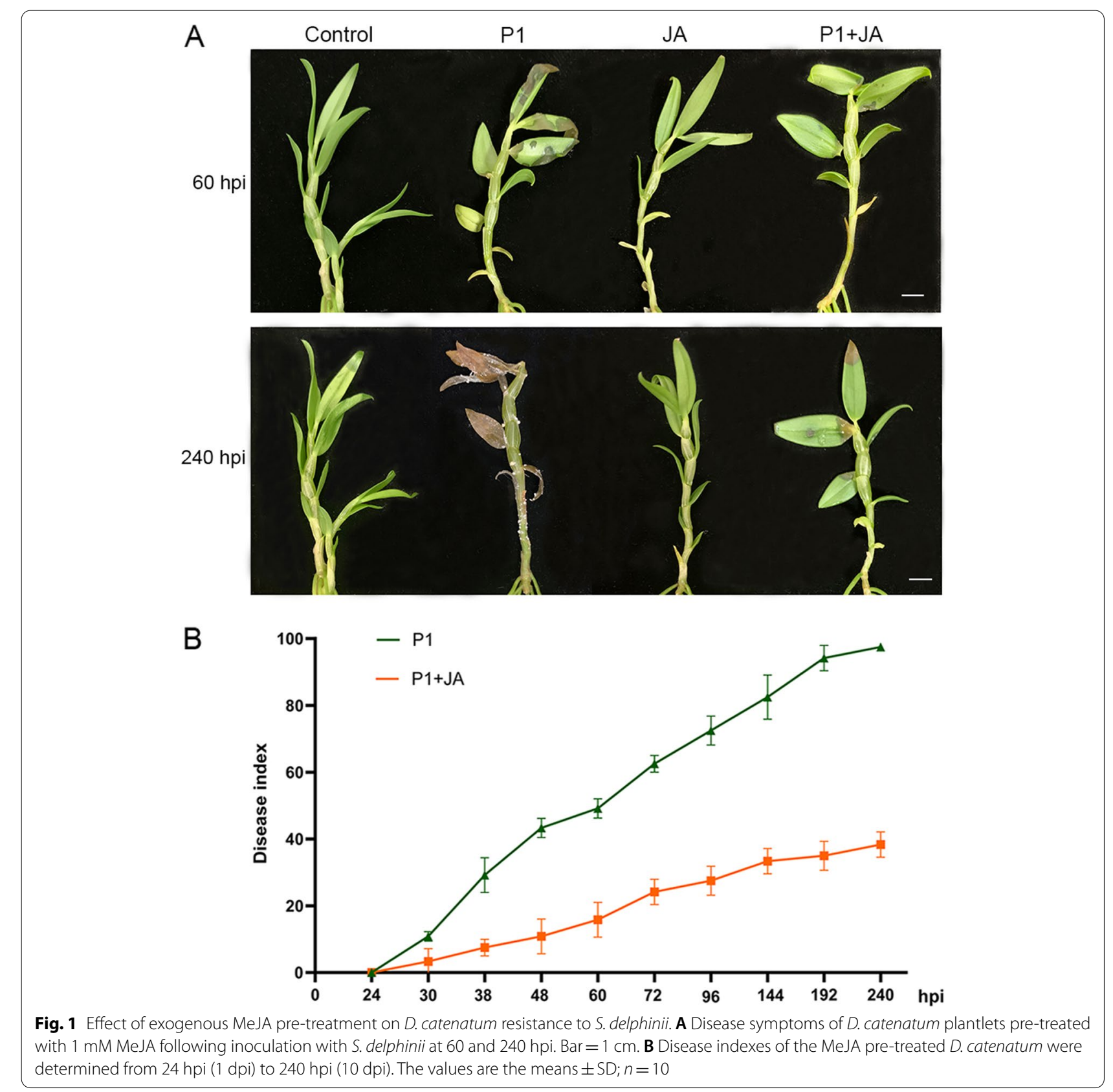


shenzhenica datasets. In total, two COI candidate protein sequences were obtained in the $D$. catenatum genome sequences, while only one was identified in the other two orchid species, respectively, suggesting that specific duplication of COI family genes happened in $D$. catenatum (Tables 1, S1, and S2). To understand the role of gene functions, subcellular localization prediction for DcCOI, $\mathrm{PeCOI}$ and $A s C O I$ genes using WoLE PSORT were performed. It was showed that $\mathrm{DcCOI}$ and $\mathrm{PeCOI}$ genes exhibited cytoplasmic localization and $A s C O I$ exhibited chloroplast localization. Moreover, the MW and pI of the COI proteins were calculated using the Protparam tool in ExPASy. The MW of COIs varied from 66,194.57 to $76,380.33 \mathrm{Da}$, and the pIs ranged from 6.31 to 8.11 . The length, MW, pI of DcCOIs and PeCOI proteins were similar, and lower than that of AsCOI, suggesting that COI protein was more conservative in epiphytic orchid.

A genome-wide search of TIFY family genes in $D$. catenatum, $P$. equestris, and $A$. shenzhenica datasets yielded 18,20 , and 13 non-redundant genes that were found to harbor the TIFY domain (Tables 1, S1, and S2). Of these TIFY domain-containing genes, 13, 14, and 7 contained both the TIFY domain and the Jas motif (PF09425) in $D$. catenatum, $P$. equestris, and $A$. shenzhenica, respectively, which were designated as JAZ proteins. The JAZ genes in $D$. catenatum and $P$. equestris were almost twice the number of $A$. shenzhenica, which is the original group of orchid [46]. It was observed that the length of the DcJAZ proteins ranged from 123 (LOC110097296) to 401 (LOC110103933) amino acid (aa) residues with an average length of $225 \mathrm{aa}$. The molecular weight ranged from $13,912.86$ to $43,134.7 \mathrm{Da}$ and the pI values varied from 5.50 to 9.34 . Subcellular location prediction showed that 10 DcJAZ proteins were localized in the nucleus. Similarly, most JAZ proteins of the other two orchid species were predicted in the nucleus, indicated that JAZ protein mainly functions in the nucleus.

After the removal of redundant gene, 12, 14, and 9 non-redundant MYC genes were identified in $D$. catenatum, $P$. equestris, and $A$. shenzhenica, respectively (Tables 1, S1, and S2). The lengths of 12 DcMYC proteins were ranged from 381 to 662 aa with an average length of 554 a. The predicted MWs of each DcMYC protein were ranged from $42,301.88 \mathrm{Da}$ to $74,540.42 \mathrm{Da}$, and the corresponding pIs were changed from 5.18 to 6.92 . The pI values of DcJAZs and PeJAZs were much lower than that of AsJAZs, showed that members of the MYC family are also relatively conservative in epiphytic orchids. The predicted subcellular location of DcMYC proteins suggested that all the $D$. catenatum MYCs were localized in the nucleus, consistent with their major roles as transcriptional factors. In addition, all these JA signal pathway genes were named after their Arabidopsis homologs.

\section{Phylogenetic and structural analyses}

To identify the classification and evolutionary patterns of COIs, TIFYs and MYCs in D. catenatum, we used COI, TIFY, and MYC proteins in A. thaliana, O. sativa, and its close relative $P$. equestris and $A$. shenzhenica as references for phylogenetic analysis, respectively.

Eight COI proteins from the above five species were clustered into three groups, COII-COIIII, and the four protein sequences from three orchid species clustered into the same group (Fig. 2A), revealing that COI protein is highly conserved in evolution among orchid species, a similar phenomenon was also observed in the members of TIFY and MYC family as mentioned later. Conserved domain analysis revealed that all the COI proteins contain typical F-box and 10-14 tandem LRR repeats (Figs. 2B and S1). DcCOI1b, PeCOI1, and OsCOI1a contained 13 LRRs with similar location distribution (Figure S1), indicating that DcCOI1b and PeCOI1 maybe harbor a similar function with OsCOI1a. The COI genes contain $2 \sim 4$ introns (Fig. 2C), which are extremely long in the three orchid species compared with those of Arabidopsis and Oryza, as attributed to a large number of repeated sequences in these three orchid genomes [46-48].

As shown in Fig. 3, TIFY proteins of the above five species were classified into eight groups (JAZI, JAZII, JAZIII, JAZIV, JAZV, TIFY, ZML, and PPD). Among them, the classification of JAZ subfamily is consistent with that of Arabidopsis [42]. As expected, the homologous genes in three orchid species always cluster together within one branch. DcTIFYs distributed in seven groups except JAZIII, indicating that the JAZIII branch was lost in the divergence from the $D$. catenatum and $P$. equestris. JAZI was the largest group with $5,6,2,10$, and 4 genes in $D$. catenatum, $P$. equestris, $A$. shenzhenica, O. sativa, and A. thaliana, respectively. The high number of JAZI genes in D. catenatum, P. equestris, and $O$. sativa is consistent with the view that significant expansion of JAZ I group genes in monocots [13], but only two members in $A$. shenzhenica indicated that the basal species in the orchid have undergone gene loss during the evolution [46]. Subsequent structural analysis focused on JAZ subfamily (Fig. 4B and C). The genomic sequences of most of these genes were less than $5 \mathrm{~kb}$, but DcJAZ6 had the longest genomic sequence of approximately $16 \mathrm{~kb}$. The clade JAZV had the longest open reading frame (ORF), whereas JAZIV clade had the shortest ORF. Insights into the phylogeny and gene structures of JAZ genes indicated that proteins that are closely related phylogenetically have similar gene structures. In addition, all of the JAZ genes had symbolic ZIM domain and Jas motif (Fig. 4B, D and E). The sequence logo for the TIFY domain (Fig. 4D), generated using all DcJAZs, showed that the D. catenatum TIFY domain was 


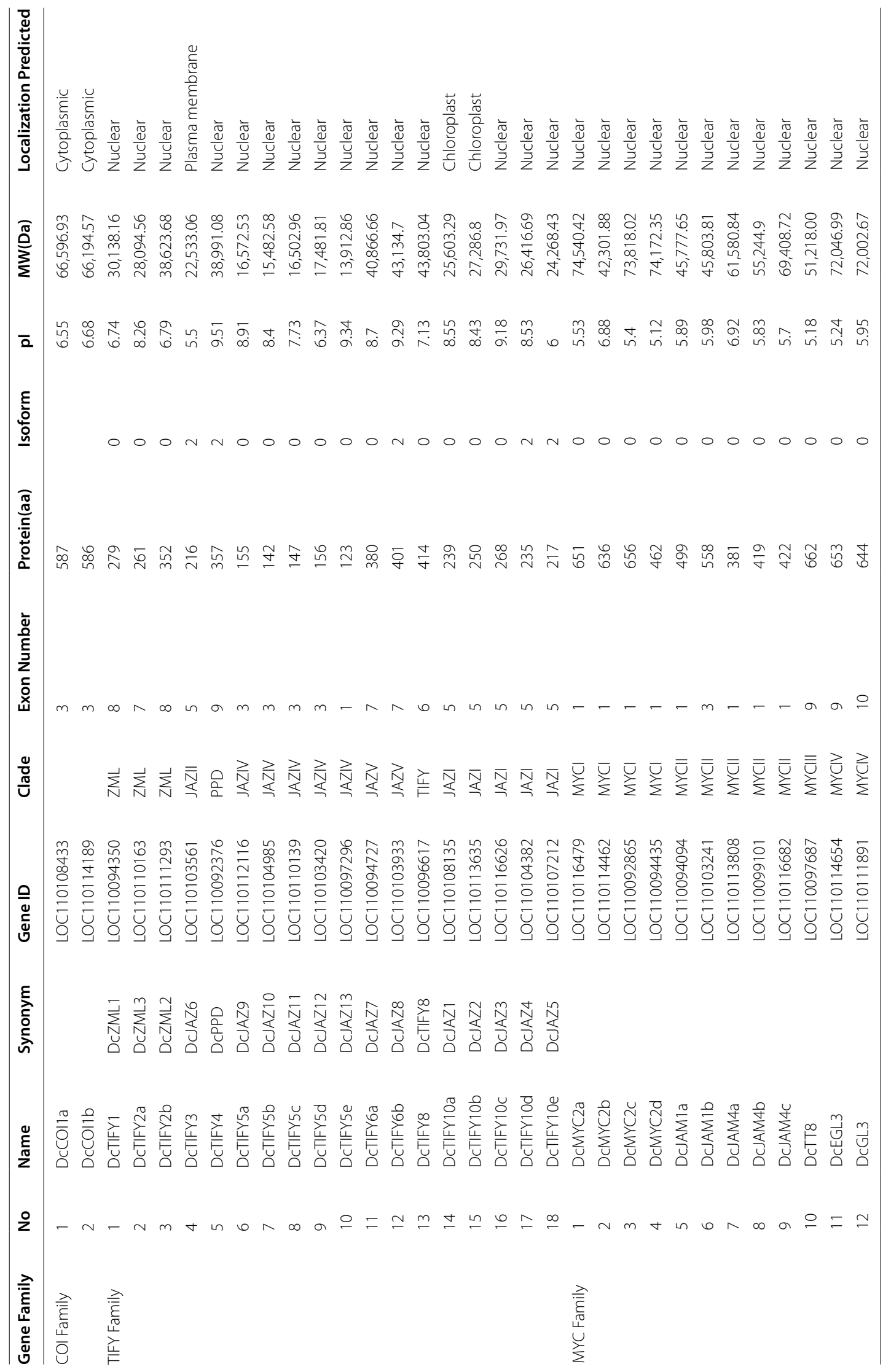



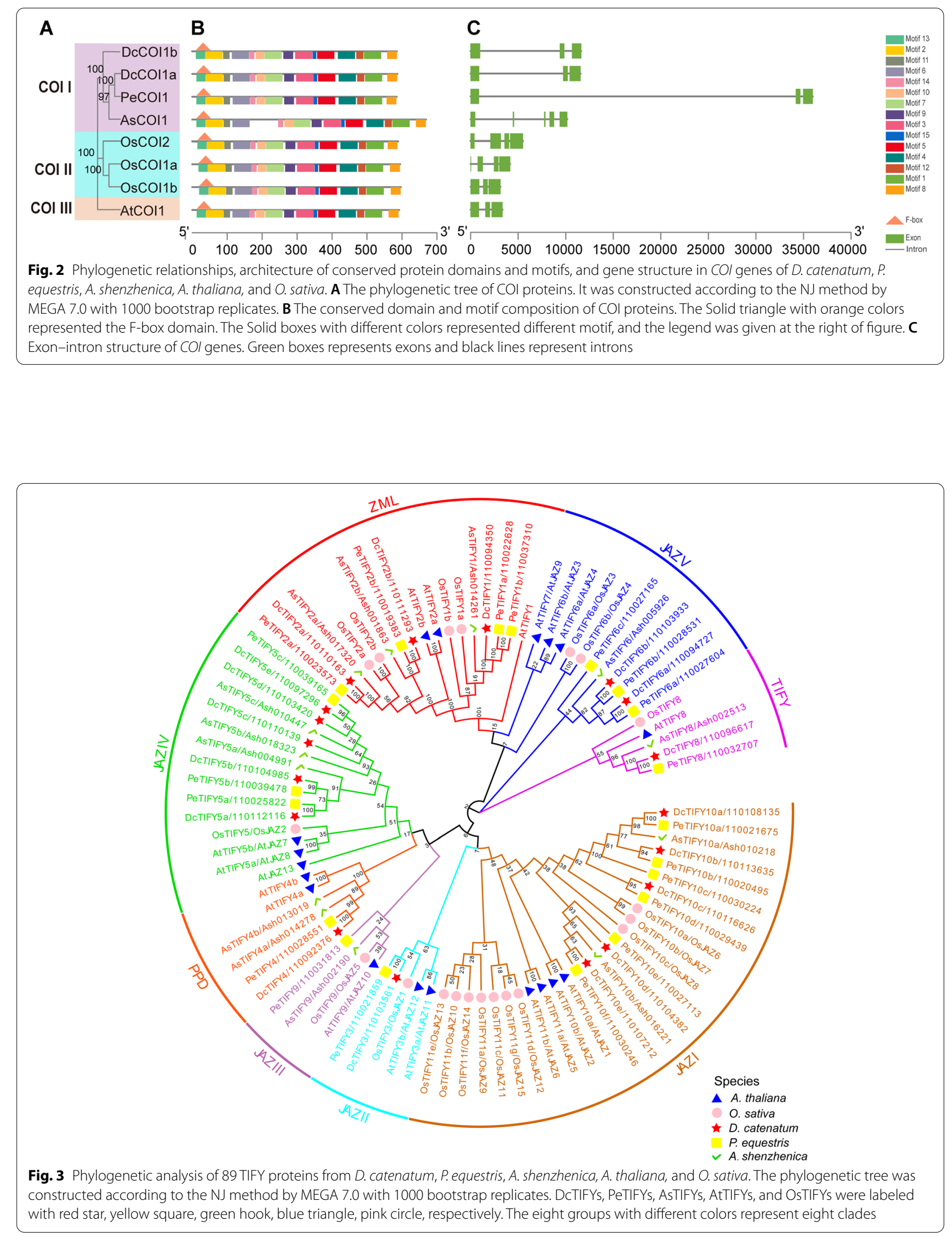


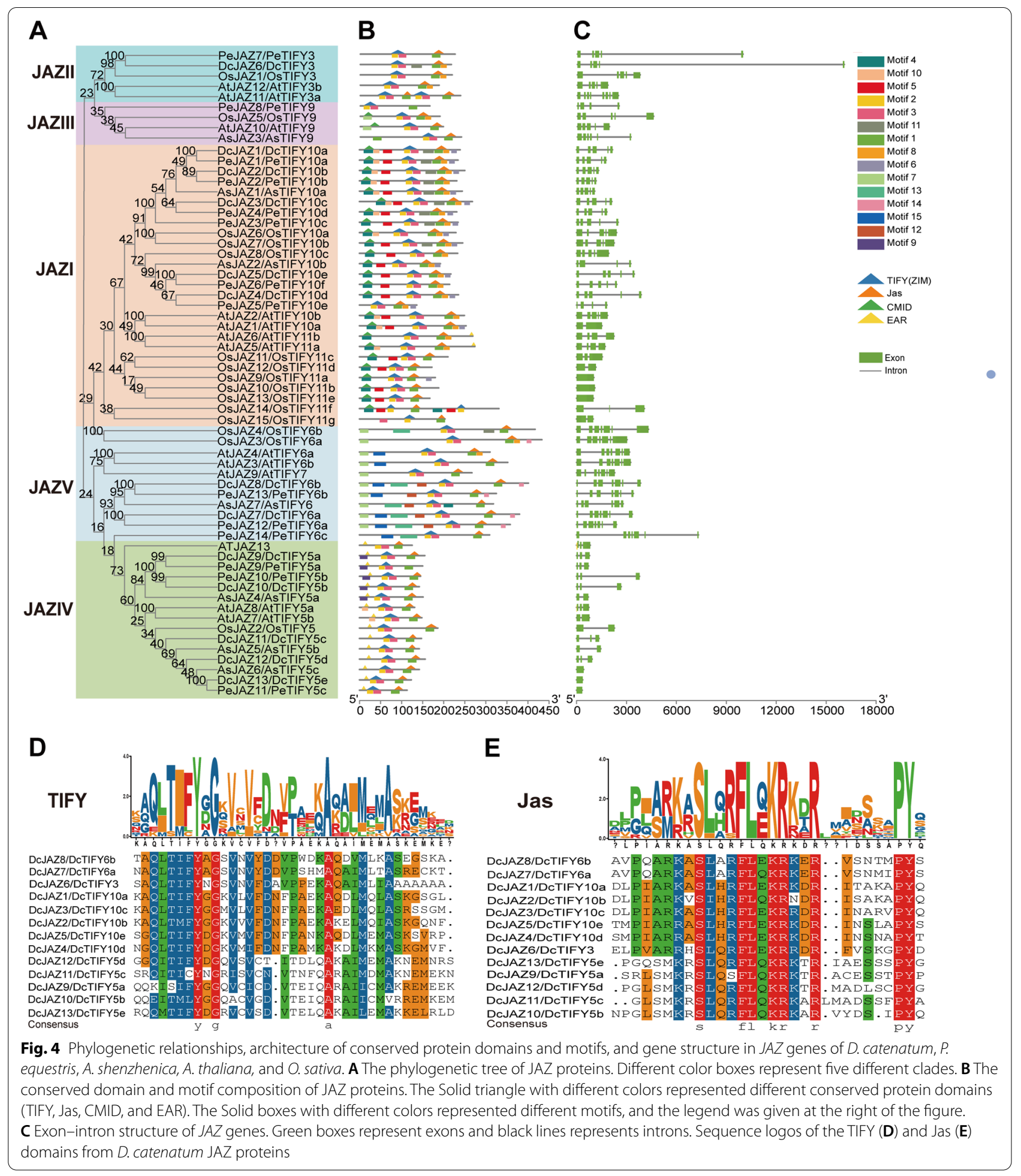

remarkably conserved and very similar to other plant species [22, 49-51]. Besides, the Jas motifs of DcJAZs display the consensus sequence $\mathrm{SLX}_{2} \mathrm{FX}_{2} \mathrm{KRX}_{2} \mathrm{RX}_{5} \mathrm{PY}$ (Fig. 4E), which involved in the interaction with $\mathrm{COI}$ and MYCs [52]. Consist with AtJAZs, the JAZIV clade proteins contained an EAR motif that mediates direct interaction with TPL without NINJA (Fig. 4B). In addition, same to AtJAZI and AtJAZIII group in Arabidopsis, DcJAZI clade showed an N-terminal CMID sequence (Fig. 4B), however, no CMID was identified in DcJAZ III 


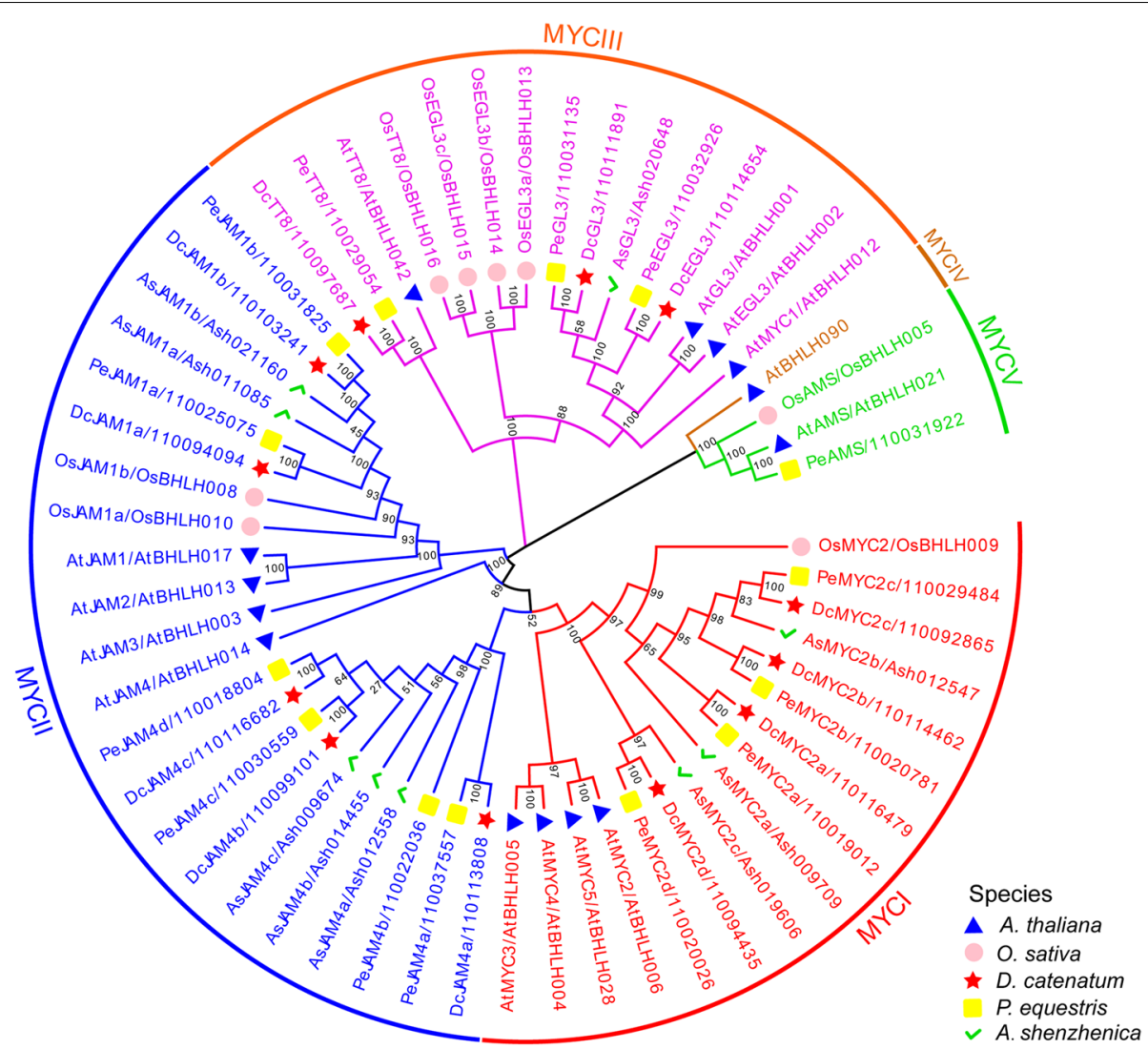

Fig. 5 Phylogenetic analysis of 57 MYC proteins from D. catenatum, P. equestris, A. shenzhenica, A. thaliana, and O. sativa. The phylogenetic tree was constructed according to the NJ method by MEGA 7.0 with 1000 bootstrap replicates. DcTIFYs, PeTIFYs, AsTIFYs, AtTIFYs, and OsTIFYs were labeled with red star, yellow square, green hook, blue triangle, pink circle, respectively. The five groups with different colors represent five clades

clade. The motif distribution was provided in Fig. 4B. JAZ genes within the same clade were usually found to share a similar motif composition, which was conspicuous in class JAZIV. Motif analysis showed conserved motif was not necessarily within a conserved functional domain. The functions of most of these conserved motifs remain to be elucidated.

A MYC family phylogenetic tree was generated using 12 DcMYCs, 14 PeMYCs, 9 AsMYCs, 8 OsMYCs, and 14 At MYCs. These MYCs were classified into five subgroups with highly bootstrap values (Fig. 5). The class MYCII was the largest, while the class MYCIV only has one member. Four, 5, and 3 DcMYCs were clustered into MYCI, MYCII, and MYCIII, respectively. However, no DcMYC was detected in MYCIV and MYCV, which may be experienced gene loss in evolution. It was worth noting that, in MYCI group, four MYC2-like proteins in D. catenatum were clustered with the other three monocots, while AtMYC2/3/4/5 were clustered in a single branch, indicating that gene replication was carried out after dividing into dicots and monocots, resulting in paralogs (Figs. 5 and 6A). There were five D. catenatum JA-ASSOCIATED
MYC2-LIKE (JAM) members in MYCII clade, which orthologs in Arabidopsis is a negative regulator of JA signaling pathway. MYCII clade members had similar motif distribution and gene structure with MYCI clade members. MYCI and MYCII genes usually consist of only one exon, significantly less than the other three clades, revealing the MYC orthologs were remarkably conserved among different species (Fig. 6C). The intron-exon structure of different DcMYC family members was diverse, while the same subclade genes were similar or same, such as MYC2-like $(D c M Y C 2 a / 2 b / 2 c / 2 d)$ and JAM4-like ( $D c J$ $A M 4 a / 4 b / 4 c)$.

\section{Tissue-specific expression patterns of core JA signaling pathway genes}

To gain insights into the potential roles of core JA pathway genes during development in D. catenatum, the published transcriptomic data were investigated in various tissues and organs, including vegetative (leaf, root, green root tip, the white part of root, and stem) and reproductive (flower bud, sepal, labellum, pollinia, and gynostemium) organs. 
According to hierarchical clustering (Fig. 7A), $D c C O I 1 a$ and DcCOI1b exhibited constitutive expressions with high levels in all tissues and organs except pollinia. Results of JAZ family members indicate that the expression profiles of $D c J A Z s$ could be split into two groups, TJ1 and TJ2 (Fig. 7B). Members of the TJ1 group (DcJAZ1/2/6/8) usually featured high expressions in most tissues. Exceptionaly, all TJ1 genes showed low expressions in pollinia, and DcJAZ1 and $D c J A Z 2$ exhibited low expression in leaf. The TJ2 genes (DcJAZ3/4/5/7/9/10/11/12/13) witnessed low expression levels in the vegetative organs, while some of these genes showed high transcriptional levels in special reproductive organs, such as DcJAZ7 in all reproductive organs except pollinia, DcJAZ3 in pollinia and gynostemium, DcJAZ12 in pollinia, and DcJAZ5/11/13 in sepal and gynostemium. These findings suggested that TJ1 genes might play essential roles in plant growth and development and TJ2 genes displayed prominent functions in flower development. The expression characteristics of MYC family genes in different tissues were quite diverse (Fig. 7C). The transcript abundance of DcMYCs could be also divided into two groups, TM1 and TM2. The majority of TM1 genes showed intermediate expressions in most of the detected tissues, whereas several expressed at high levels in specific tissues, such as DcGL3 in leaf, $D c J A M 4 b$ and DcEGL3 in gynostemium, DcMYC2c in pollinia and gynostemium, DcJAM1a in sepal and gynostemium, DcJAM $1 b$ and $D c M Y C 2 b$ in pollinia, suggesting that these genes might play essential roles in specific tissue and organ development. TM2 genes (DcMYC2d, DcJAM4a, DcJAM4c, and DcTT8) showed low expressions in most tissues. However, $D c M Y C 2 d$ are highly expressed in sepal and gynostemium, illustrating it might perform a dominant function during sepal and gynostemium development.

\section{Transcriptome sequencing}

To gain further insights into the role of exogenous MeJA application in the enhanced resistance to $S$. delphinii in $D$. catenatum, a comparative transcriptome analysis was performed. Four leaf samples were obtained at $24 \mathrm{~h}$ after inoculated with sterile distilled water (mock) or S. delphinii (P1) from plantlets pre-treated with 0 or $1 \mathrm{mM}$ MeJA (CK, P1, JA, and P1+JA), respectively. All samples used for transcriptome sequencing have three biological replicates (Table S3). Using the HiSeq4000 sequencing platform, 107.83-148.06 million raw reads were generated for each RNA sample. Based on the $D$. catenatum genome, the number of mapped clean reads was 47.31 69.65 million (Table S4). Comparison of the transcript abundances in the leaves of CK, P1, JA, and P1 + JA, 5255 differentially expressed genes (DEGs) were detected (Table S5). Comparison analysis of the plantlets inoculated with $S$. delphinii pre-treated by MeJA $(\mathrm{P} 1+\mathrm{JA})$ and those only MeJA pre-treated (JA), 982 DEGs were identified, with 825 significantly upregulated and 157 significantly downregulated genes. Comparing the control plantlets and MeJA-pretreated plantlets after S. delphinii inoculation (P1 vs P1+JA) uncovered 848 genes significantly up-regulated in the latter (Table S5).

\section{JA pathway genes significantly responds to S. delphinii}

Comparative transcriptome changes for genes involved in JA pathway response to pathogen and MeJA were investigated (Table S6). Figure 8 shows the S. delphinii (P1) and MeJA-induced expression pattern changes of 31 related genes in this pathway. Gene expression levels were illustrated using a heatmap and estimated by log scale. The transcription levels of the two DcCOI1 genes did not shown significantly changes after MeJA treated or S. delphinii infected (Fig. 8 and Table S6). This result may be attributable to the fact that COI1 as JA receptor is functionally regulated mainly in protein level but not in transcription level. Most of the DcJAZ genes were significantly upregulated in three treatment samples (P1, JA, P1+JA) compared with the control (CK). The expression pattern of these JAZ genes can be divided into four categories (Fig. 8). The PJ1 class genes (DcJAZ1/2/5/6/13) were significantly induced by MeJA or $S$. delphinii, respectively, and the expression had an additive effect when MeJA and S. delphinii were both treated; the PJ2 class genes (DcJAZ4/11/12) were significantly upregulated by MeJA or $S$. delphinii respectively, but it was more significantly response to $S$. delphinii

\footnotetext{
(See figure on next page.)

Fig. 6 Phylogenetic relationships, architecture of conserved protein domains and motifs, and gene structure in MYC genes of D. catenatum, $P$. equestris, A. shenzhenica, A. thaliana, and O. sativa. A The phylogenetic tree of MYC proteins. Different color boxes represent five different clades. B The conserved domain and motif composition of MYC proteins. The Solid triangle with orange and light blue represented bHLH domain and bHLH-MYC_N domain, respectively. The Solid boxes with different colors represented different motif and the legend was given at the right of figure. C Exon-intron structure of MYC genes. Green boxes represent exons and black lines represent introns. D Sequence alignment of JID, TAD, basic, and HLH domain of DcMYC2a, DcMYC2b, DcMYC2c, DcMYC2d, AtMYC2, AtMYC3, AtMYC4, and AtMYC5 proteins. Sequence alignment was performed using ClustalX. Red triangles represented amino acid residues involved in the AtMYC3-JAZ interaction. Blue triangles represented residues that are related to specific DNA recognition in MYC2. Orange triangles and black triangles represented residues that are required for MYC2 dimer formation and tetramer formation, respectively
} 


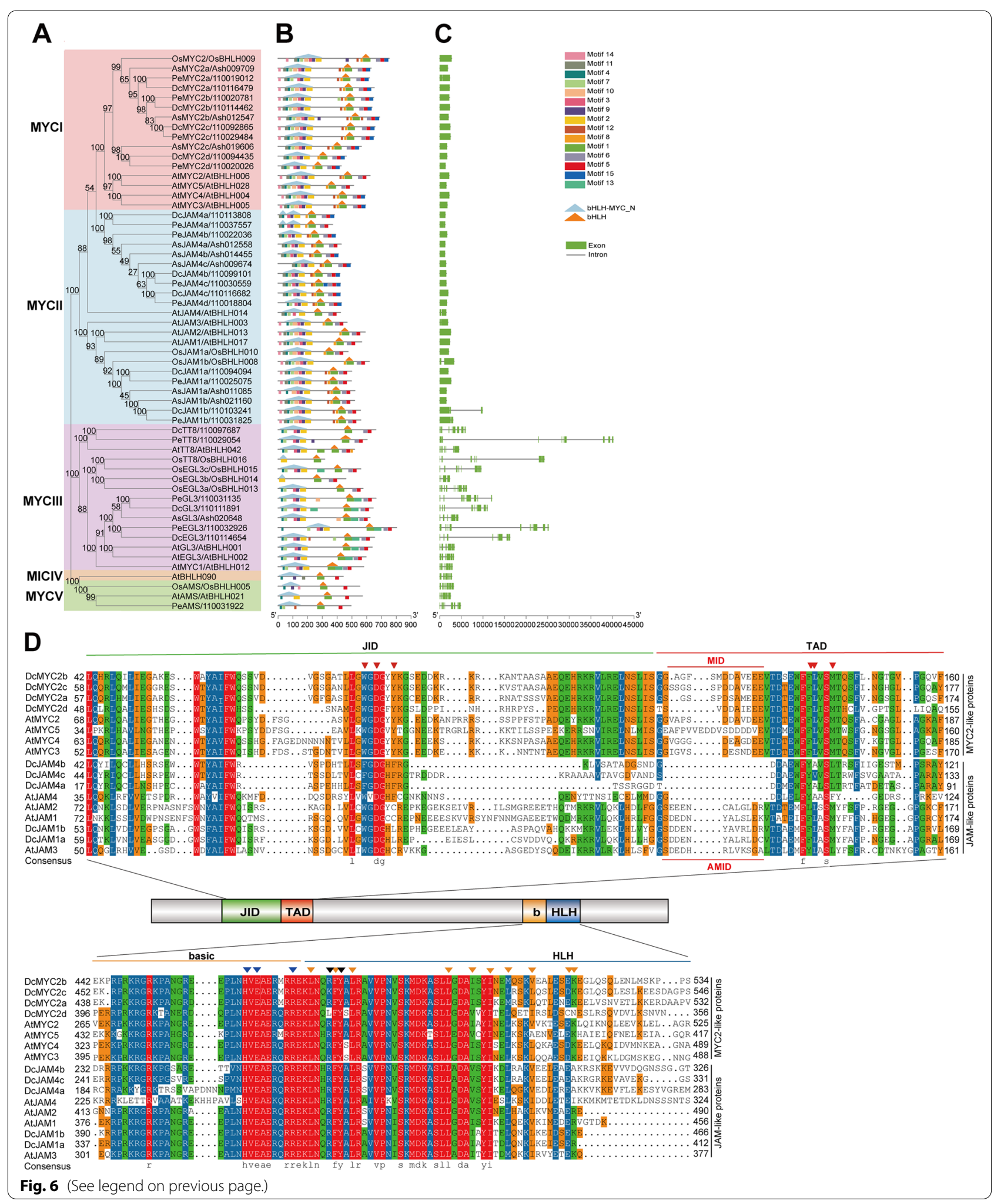




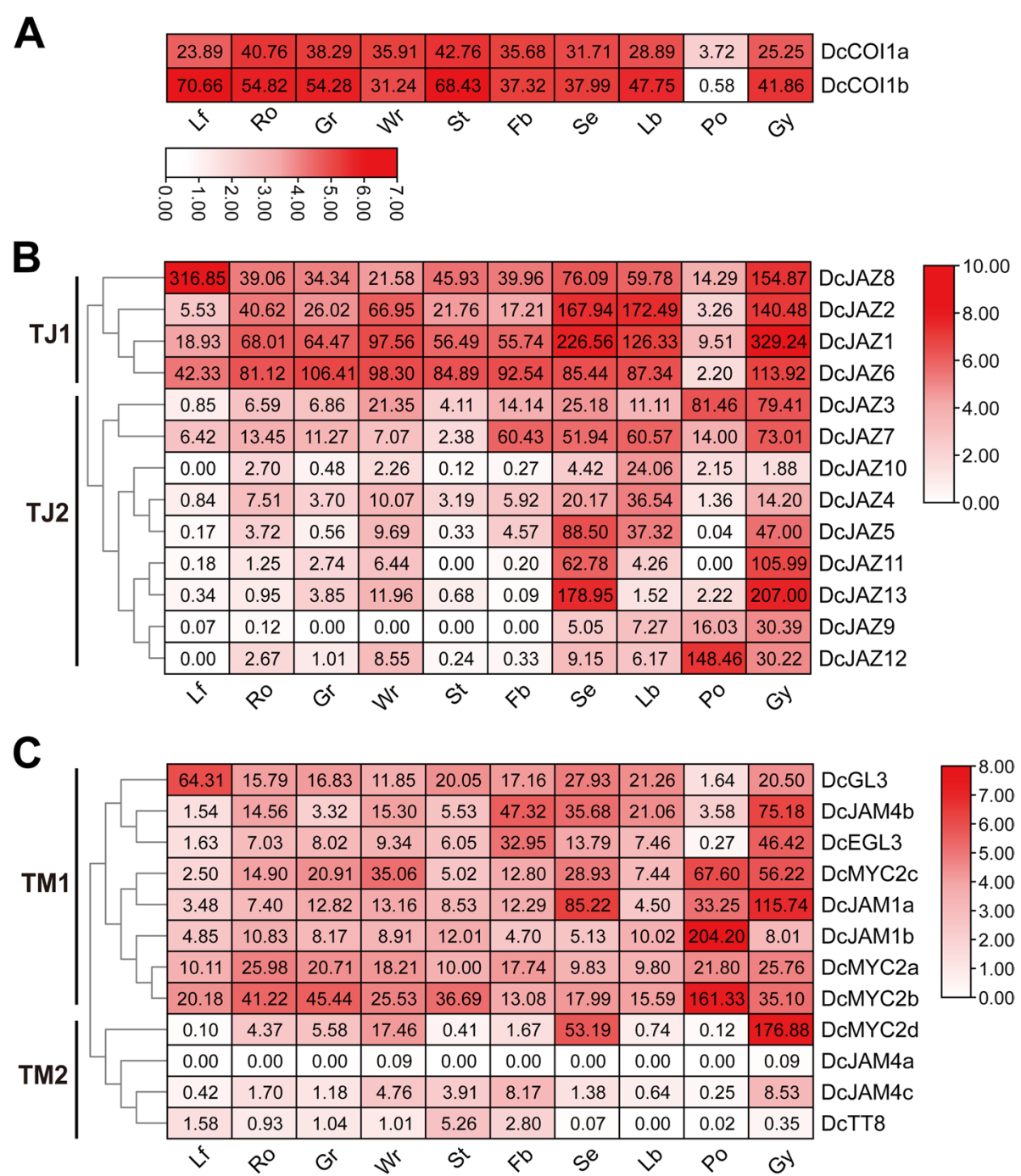

Fig. 7 Expression profiles of JA signaling pathway genes in different tissues and organs in D. catenatum. A Expression patterns of DcCO/genes in ten $D$. catenatum tissues and organs. B Expression patterns of DCJAZ genes in ten D. catenatum tissues and organs. C Expression patterns of DcMYC genes in ten D. catenatum tissues and organs. Lf: leaves, Ro: roots, Gr: green root tip, Wr: the white part of roots, St: stems, Fb: flower buds, Se: sepals, Lb: labellum (lip), Po: pollinia, and Gs, gynostemium (column). The $\log _{2}$ transformations of the expression values were used to generate the heat map with TBtools software

than MeJA. However, there was no evidently expression difference between $\mathrm{P} 1$ and $\mathrm{P} 1+\mathrm{JA}$, indicating that these genes are responsive to $S$. delphinii but not MeJA; the PJ3 class genes (DcJAZ3/8/9/10) were only induced by MeJA, suggesting that these type genes did not respond to $S$. delphinii, and may be involved in the growth and development process related to JA pathway; the fourth type of genes (DcJAZ7) was not detected obvious transcriptional changes. The expression of MYC family genes was more complicated, and its expression profiles could also be divided into four types (Fig. 8). The expression patterns of PM1
(DcMYC2b and DcGL3) and PM3 (DcJAM1a) group were consistent with PJ1 and PJ2 classes of $J A Z$ genes, respectively. The PM2 $(D c M Y C 2 c / 2 d)$ class genes showed that $S$. delphinii inhibited but MeJA significantly induced expression, indicating that this type of genes was a negative regulator in $D$. catenatum response to $S$. delphinii; the PM4 class genes were significantly downregulated in samples P1, JA, and $\mathrm{P} 1+\mathrm{JA}$, which was consistent with their role as negative regulators of JA pathway. However, JA-responsive genes, DcPR3 (LOC110093420) and DcLOX2 (LOC110106362) showed significantly induced in 


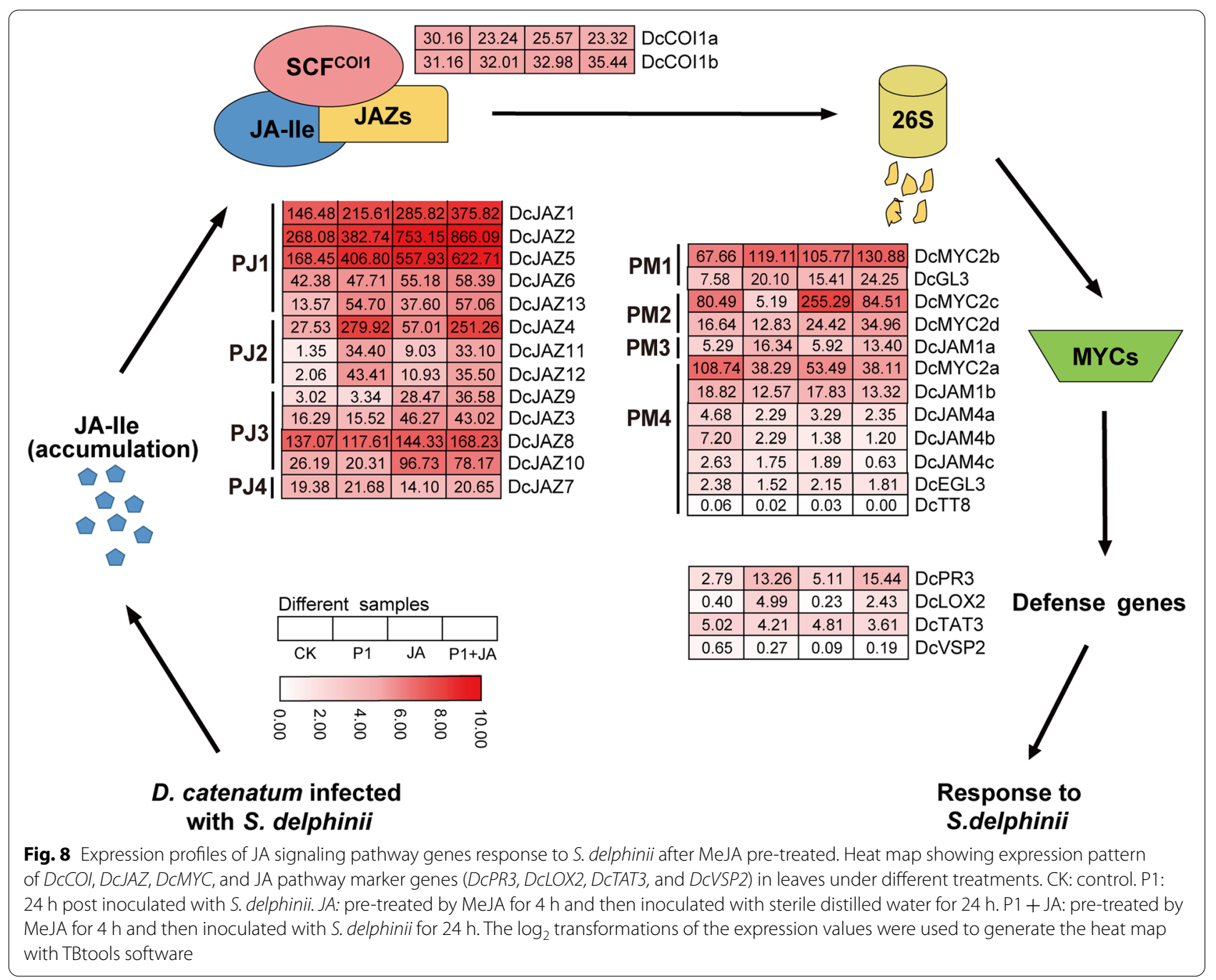

$\mathrm{P} 1$, JA, and P1 + JA samples, but with lower expression levels. DcTAT3 (LOC110109169) and DcVSP2 (LOC110107086) exhibited weak induction.

Real-time quantitative PCR of 13 representative genes confirmed the RNA-sequencing data (Fig. 9). DcCOI1a and $D c C O I 1 b$ had no markedly expression changes. $D c J A Z 1, D c J A Z 2$ and DcJAZ5 were tightly co-expressed with $D c M Y C 2 b$, with their transcript levels significantly upregulated in $\mathrm{P} 1, \mathrm{JA}$, and $\mathrm{P} 1+\mathrm{JA}$ treatment samples. DcJAM4b showed negative regulation, while the transcript levels of DcJAZ4 and DcJAM1a were high in P1 and P1+JA samples but low in JA treated sample. DcPR3 and DcLOX2 were significantly induced in three treated samples. $D c M Y C 2 c$ induced by JA but not seriously respond to $S$. delphinii, while $M Y C 2 c$ significantly downregulated after P1 treatment in transcriptome data. The DcJAZ8 was significantly upregulated in $\mathrm{P} 1+\mathrm{JA}$, which was inconsistent with transcriptome expression data.

\section{Discussion}

Southern Blight is one of the most destructive fungal diseases against $D$. catenatum planting, causing almost no yield [5]. However, an understanding of the mechanism(s) underlying $D$. catenatum resistance to $S$. delphinii has not been reported. After decades of research, JA has been firmly established as a central role in mediating defense response and facilitate the adaptation of plants to a wide range of biotic and abiotic stresses, especially in defenses to necrotrophic pathogens and chewing insects [12, 42]. In this study, $D$. catenatum plantlets pre-treated with MeJA obviously alleviated $S$. delphinii infection symptoms, indicating that the MeJA was useful for enhancing D. catenatum resistance to $S$. delphinii (Fig. 1). 


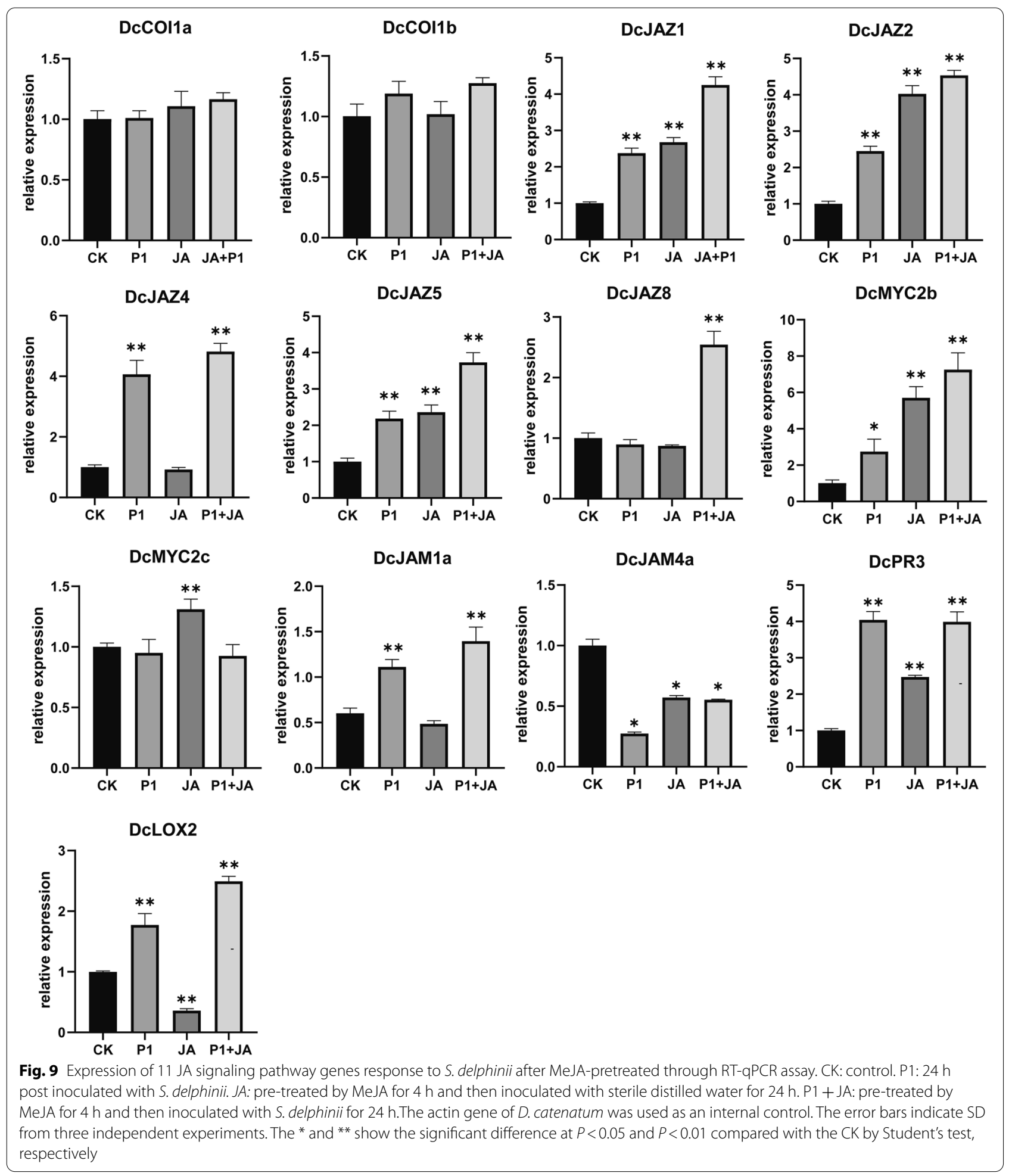

The F-box protein COI1 was isolated in 1990s, which is the first milestone in JA signaling pathway $[22,53]$. Both JA related pathways are depend on the COI1 gene. In Arabidopsis, a single COI1 (At2939940) gene was characterized and coil mutant exhibits male sterile, resistant to a coronatine-producing strain of pseudomonas syringae, insensitive to MeJA, and sensitive to $B$. cinerea $[53,54]$. In the rice genome, three 
COI homologs were identified [55], i.e., OsCOI1a (Os01g0853400), OsCOI1b (Os05g0449500), and OsCOI2 (Os03g0265500). OsCOI1a and OsCOI1b share over $80 \%$ amino acid sequence similarity, while OsCOI2 shows fewer sequence identities (63\%) with the two OsCOI1 proteins. Moreover, OsCOI1 RNAi lines, targeting a sequence conserved in OsCOIIa and OsCOI1b, show altered plant height, internode length, grain length, and increased susceptibility to herbivores [56, 57]. Here, 2 COI1 genes were identified in D. catenatum. Compared with Arabidopsis, the number change of COI genes in $D$. catenatum may attribute to undergone two wholegenome duplication (WGD) events (a T WGD shared by most monocots and a recent WGD specific to the Orchidaceae lineage $[46,58]$. The gene structure and motif distribution of the pair of DcCOI1 genes, DcCOI1a/1b, were conserved. Moreover, $D c C O I 1 a / 1 b$ expressed in all examined tissues with similar expression patterns (Fig. 8), indicating that they might have experienced subfunctionalization [59]. It is intriguing that $D c C O I 1 a / 1 b$ expressed in pollen were significantly lower than the other tissues. Previous study found that COI performs functions at the protein level and a proper protein level of COI1 is important to maintain distinct JA responses [60].

The plant-specific group JAZ proteins plays a pivotal role in MYC TFs activity change and has emerged as nodes for signal crosstalk in hormone response pathways. Arabidopsis genome contains 13 JAZ members that are classified into five groups (I-V) [42]. In the present study, 13 DcJAZ proteins were identified and classify based on the homology with that of Arabidopsis (Fig. 4A and Table 1). However, no DcJAZs were found in JAZIII group. This result is inconsistent with the view that all the five groups exist in all angiosperms [13]. We speculated that group III genes might have been experienced specifically lost during the evolution of $D$. catenatum. The domain architecture of JAZI group proteins (DcJAZ1-5) was more similar to AtJAZ1 and AtJAZ2, containing a CMID domain but no EAR domain [42]. Among them, DcJAZ1/2/5 were simultaneously significantly induced by P1, JA, and both (PJ1 expression mode, Fig. 8), indicating that these three $D c J A Z s$ played a critical part in enhancing the resistance to $S$. delphinii in D. catenatum. DcJAZ4 exhibited specific significantly respond to $S$. delphinii but weaker respond to MeJA treatment (PJ2 expression mode, Fig. 8), indicating that DcJAZ4 acted more as a hub to interact with other pathways (ethylene-JA, salicylic acid (SA)-JA etc.) to enhance the defense responses to pathogens in D. catenatum. DcJAZ3 showed PJ3 expression pattern and was significantly induced by JA instead of S. delphinii, speculating that DcJAZ3 might be involved in JA-mediated growth and development pathways. The single member of JAZII group, DcJAZ6, exhibited PJ1 expression pattern but no significant changes detected. Its Arabidopsis homologous gene mutant jaz12-1 shows normal JA responses and performs a redundant function in the JA response. Therefore, DcJAZ6 might function redundantly with $D c J A Z 1 / 2 / 5$ in $D$. catenatum. The JAZIV group with five members had the shortest protein length in all the five groups (Fig. $4 \mathrm{~B}$ and Table 1). Its close Arabidopsis homologues AtJAZ7, AtJAZ8, and AtJAZ13 harbors a divergent Jas motif that interacts weakly with COI1 but is able to interact with MYC2, and behaves as a constitutive repressor in the jasmonate pathway [25, 32, 33]. The DcJAZs in this group showed three expressing patterns (PJ1, PJ2, and PJ3), meaning they functions in different JA pathways act as inert JAZ proteins. The JAZV group members (DcJAZ7 and DcJAZ8) showed different structures with the other four groups with the longest protein length and the highest number of exons. In Arabidopsis, group JAZV proteins includes AtJAZ3, AtJAZ4, and AtJAZ9 [42]. Atjaz9-1 mutant has normal JA-mediated responses but a specific role in the JA-GA crosstalk, acting functional redundancy in JA responses [33, 61]. The knockout lines Atjaz4-1 were hypersensitive to JA-induced leaf senescence [62]. Combined with the expression profile of DcJAZ7 (no obvious change) and DcJAZ8 (significantly response to MeJA but not $S$. delphinii), suggesting that these two genes mainly participated in JA-mediated growth and development in D. catenatum. All in all, further in-depth molecular dissection of DcJAZ1, DcJAZ2, and DcJAZ5 will enrich the defense mechanisms of $D$. catenatum response to $S$. delphinii.

MYC2 and its closely related paralogs MYC3, MYC4, and MYC5 orchestrated transcriptional reprogramming, which is a central theme of JA pathway [27, 63]. Although the molecular components (COI1/JAZ/MYC2 complex) of JA signaling pathway in plants are relatively conserved, but MYC2 regulates downstream genes is species-specific [31, 37]. In Arabidopsis, MYC2 differentially regulates the expression of two groups of JA-induced genes. MYC2 activates NAC019 further positively regulates wound response genes (first group) but inhibits the Ethylene-Response-Factor1 (ERF1) to further negatively regulates pathogen response genes (second group) [64]. Arabidopsis myc2/jin1 mutant shows increased resistance to $B$. cinerea and $P$. cucumerina, and sensitivity to insect pests [12, 29]. In tomato, MYC2 positively regulates both of these two group genes by activating JA2L and ERF.C3 transcription factors, respectively, and the necrosis area of the tomato MYC2-RNAi line inoculated with $B$. cinerea was significantly larger than those of the wild type [31]. Arabidopsis MYC2 and its tomato homolog have distinct action modes. It might be due to recruiting different partner proteins in transcriptional regulatory activities of pathogen 
response genes [31, 45]. In this study, we identified 12 MYC members with bHLH domain and bHLH-MYC_N domain and classified them into five groups. MYCI group genes $(D c M Y C 2 a / 2 b / 2 c / 2 d)$ had close homology with AtMYC2/3/4/5. The domain architecture and gene structure of DcMYC2a, DcMYC2b, and DcMYC2c were similar, but shorter protein lengths with compact domain architecture was found in DcMYC2d, which consistent with that DcMYC2a, DcMYC2b, and DcMYC2c had a more closely phylogenetic genetic relationship. However, the expression patterns of these four duplicated $D c M Y C 2$ genes were dramatic differences. DcMYC2a exhibited PM4 pattern with negative regulation under both MeJA and S. delphinii. DcMYC2c and DcMYC2d showed upregulated by JA, but repressed by S. delphinii, suggesting these two genes might involve in JA-regulated growth and development pathways. DcMYC2b showed PM1 expression pattern and were positively regulated by MeJA and S. delphinii, suggesting that MYC2b may act as positive regulator and play a key role in JA-mediated defense to $S$. delphinii stress in D. catenatum. We also detected the expression level of MYC2-regulated pathogen defense genes (AtPDF1.2 orthologous gene in D. catenatum was not found), DcPR3, DcLOX2, DcTAT3, and DcVSP2. Transcriptome results showed that DcPR3 and $D c L O X 2$ significantly response to $S$. delphinii, which was validated in qRT-PCR analysis (Figs. 8 and 9). Therefore, we speculated the action mode of $D c M Y C 2 b$ was same to tomato $M Y C 2$. It worth further investigate its direct targets by high-throughput ChIP-seq method or yeast onehybrid system. In MYCII group, five JAM genes, termed the MYC2-TARGETED BHLH1 (MTB) genes in tomato [37], were isolated. In Arabidopsis, the bHLH subclade IIId proteins, bHLH17/JAM1, bHLH13/JAM2, bHLH3/ JAM3, bHLH14/JAM4, were found to compete with MYC2-like TFs by binding to its target gene promoters $[37,65,66]$. MYC2-like TFs interacts with the JAZ protein through the $\mathrm{N}$-terminal JID domain. Adjacent to JID is the transcriptional activation domain (TAD), which is responsible for the interaction with MED25. MED25 is a subunit of the mediator complex, which recruits general transcription machinery such as RNA polymerase II to achieve the transcription factor activity of MYC and initiate its transcription of target genes. The MID region in the TAD region is necessary for the interaction between MYC2 and MED25. JAMs-like TFs have a normal JID domain and an altered MID domain (AMID) and can interact with JAZ but cannot bind to MED25 [37]. Same to Arabidopsis, the MID-corresponding region of DcJAMs had been altered (Fig. 6D). Sequence alignment indicated that the MID was generally conserved in MYC2-like proteins and that the AMID was conserved in JAMs-like proteins in Arabidopsis and D. catenatum.
Notably, there was some sequence divergence in AMID domain between DcJAM4s and the other DcJAMs-like proteins and their functional divergence is worthy to further investigation. Transcriptome analysis revealed that four of this group genes $(D c J A M 1 b / 4 a / 4 b / 4 c)$ were downregulated after $S$. delphinii, MeJA, and both treatment, which was consistent with the role as the negative regulators of JAMs-like TFs. However, DcJAM1a showed a significantly improved expression response to S. delphinii, suggesting a distinct regulated mechanism.

Based on these observations, we propose a model of $D$. catenatum JA signaling pathway response to S. delphinii. Upon $S$. delphinii elicitation, $D$. catenatum elevated JAIle levels promote $\mathrm{SCF}^{\mathrm{DcCOI1} / 1 \mathrm{~b}}$-dependent degradation of DcJAZ1/2/5 repressors, thereby DcMYC2b rapidly and directly regulates the transcription of downstream DcMYC2b-targeted TFs (DcMTFs), which in turn regulate the expression of wounding-responsive (DcPR3) or pathogen-responsive genes ( $D c L O X 2$, Figure S2). Further functional study of the DcMTFs will help to clarify this issue.

\section{Conclusion}

In this study, pre-treated with $1 \mathrm{mM}$ MeJA significantly improved the resistance of $D$. catenatum to $S$. delphinii. Based on this phenotype, we identified 2 COI1, 13 JAZ, and 12 MYC proteins involved in core JA pathway of $D$. catenatum. These core JA pathway members of D. catenatum were classified and their evolutionary relationships with Arabidopsis, rice, and its closely related orchid species $P$. equestris and $A$. shenzhenica, were evaluated depend on phylogenetic relationship and substrate specificity. The expression profiles of $D$. catenatum core JA pathway genes in different tissues and organs was further analyzed. Comparative transcriptome analysis of the core JA pathway genes in $D$. catenatum planets under MeJA, S. delphinii, and combined treatments revealed that $D c J A Z 1 / 2 / 5$ and $D c M Y C 2 b$ synergistically promote JA mediated defense response in $D$. catenatum. Therefore, further studies are required to understand how $D c J A Z 1 / 2 / 5$ and $D c M Y C 2 b$ is regulated and whether and how it regulates other genes (DcMTFs) to control $D$. catenatum responds to $S$. delphinii. Our findings may help establish available avenues for plant breeding strategies aimed at southern blight disease resistance via JA-mediated enhanced plant immunity.

\section{Methods \\ Biological materials}

D. catenatum clonal cultivar 'Jingpin xianshi' (6A2B) is susceptible to $S$. delphinii, which was obtained by subculture from stem explants, placed in a modified $1 / 2$ Murashige and Skoog (MS) medium [67], and cultivated 
in a growth room with a $12 \mathrm{~h}$ light/ $12 \mathrm{~h}$ dark photoperiod, $60 \%$ relative humidity, and $25{ }^{\circ} \mathrm{C}$ temperatures. The seedlings used for experiments were in same growth status and about $5 \mathrm{~cm}$ high, 6 leaves and $3 \sim 5$ roots, and the stem diameter was about $0.3 \mathrm{~cm}$.

\section{MeJA treatment}

To study the effects of MeJA on D. catenatum plantlets, four-month-old plantlets were spotted with $0.25 \%$ ethanol solution (control) or a solution containing $1 \mathrm{mM}$ MeJA (Sigma-Aldrich, 392,707) prepared in $0.25 \%$ ethanol solution (1 mL of per plantlet). After MeJA pre-treatment for $4 \mathrm{~h}, D$. catenatum plantlets were inoculated with S. delphinii (named P1), which was isolated and identified in our laboratory [5].

\section{Pathogen challenge assays}

S. delphinii grew on PDA medium at $25{ }^{\circ} \mathrm{C}$ for 6 days. Five $5 \mathrm{~mm}$ agar disks containing mycelia were collected and cultured in $200 \mathrm{~mL}$ PDB medium for 5 days at $25^{\circ} \mathrm{C}$ with shaking (180 rpm).

Four-month-old $D$. catenatum plantlets pre-treated by MeJA or $0.25 \%$ ethanol solution as indicated above were sprayed with mycelia suspensions $(2 \mathrm{~mL}$ per plantlet). Control plantlets in each treatment were inoculated with an equal volume of sterile distilled water. S. delphinii bioassays were performed at $28{ }^{\circ} \mathrm{C}$ under a photoperiod of $12 \mathrm{~h}$ light/12 h dark.

\section{Disease assessment after S. delphinii inoculation}

The progress of $S$. delphinii infection was followed for 10 days by observing the development of necrosis in the infected leaves, which can be detected at $24 \mathrm{~h}$ post inoculation (hpi), and infection ratings were assigned to the inoculated plantlets: 0 , no necrosis; 1 , one or two leaves showing a small part of necrosis lesion; 2, one or two leaves showing a large area of necrosis lesion; 3 , over three leaves showing necrosis and some leaves dropped off; 4, dead/decayed plant [68]. At least 10 plantlets were inoculated in each experiment. Experiments were repeated at least three times with similar results. The disease index was calculated with the following formula: disease index $=((\Sigma$ disease grade $\times$ the number of infected plantlets $) /($ total assessed plantlets $\times 4)) \times 100$. Photographs were taken at 60 and $240 \mathrm{hpi}$.

\section{Genome-wide identification of core JA signaling pathway} genes in some representative orchid species

All the COI, TIFY, MYC sequences including genomic DNAs, coding sequence (CDS), and proteins in A. thaliana and Oryza sativa were downloaded from the plant genomics resource Phytozome v12 (http://phytozome. jgi.doe.gov/pz/portal.html). D. catenatum, P. equestris and $A$. shenzhenica genome sequences were retrieved from NCBI Genome (https://www.ncbi.nlm.nih.gov/ genome/). To acquire the COI domain-containing sequences in $D$. catenatum, $P$. equestris, and $A$. shenzhenica, the protein sequences of $A$. thaliana [22] and O. sativa [69] were used as queries to search homologs against NCBI database using BLASTp tool. The hidden Markov model (HMM) profile of TIFY domain (Pfam accessions: PF06200) was downloaded from the Protein family (Pfam) database (https://pfam.xfam.org/) [70], and used as queries to search for potential TIFY proteins in the $D$. catenatum, P. equestris and $A$. shenzhenica protein datasets by using HMMER3.0 [71] with an E-value cutoff of 1.0E-05. The MYC family protein was filtered for Pfam database identifiers of the bHLH domain (PF00010) and bHLH-MYC_N domain (PF14215), respectively. All the hits were further confirmed to remove the incomplete and redundant sequences.

The acquired sequences were submitted to ExPASy (https://web.expasy.org/protparam/) to calculate the molecular weights (MW) and theoretical isoelectric points (pI). Subcellular localization were predicted using the WoLF PSORT (https://www.genscript.com/wolf-psort.html).

\section{Gene structure, domain architecture, conserved motif, and phylogenetic relationship analyses}

The conserved motifs were investigated by MEME version 5.3 online tool (http://meme-suite.org/tools/meme). The domain organization was analyzed using the Simple Modular Architecture Research Tool (SMART) and NCBI CD search program. The gene structure, protein domain architecture, and motif composition was visualized using the TBtools [72].

Multiple sequence alignment of COI, TIFY, and MYC proteins was performed using ClustalX [73] with default parameters, respectively. Neighbor-joining (NJ) trees were constructed using MEGA7 [74] with a pairwise deletion option for gaps, p-distance method, and bootstrap test of 1000 replicates. The phylogenetic tree was subsequently visualized with EvolView (https://evolg enius.info/evolview-v2/\#login).

\section{RNA-seq analysis}

The leaf samples of $D$. catenatum plantlets were pretreated with 0 (control) or $1 \mathrm{mM}$ MeJA were harvested for transcriptome sequencing at $24 \mathrm{hpi}$. Three independent RNA samples (biological replicates) were performed for each treatment. RNA was extracted using the MiniBEST Plant RNA Extraction Kit (TaKaRa, Japan) according to the manufacturer's instructions. The paired-end sequencing was performed on an Illumina Hiseq4000. 
The Illumina RNA-seq data generated from the different tissues were downloaded at the NCBI Sequence Read Archive (SRA) provided by Zhang et al. [46]. SRA data of ten tissues in an individual of wild $D$. catenatum, including leaf (SRR4431601), stem (SRR4431600), root (SRR5722140), green root tip (SRR4431599), white part of the root (SRR4431598), flower bud (SRR4431603), sepal (SRR4431597), labellum (SRR4431602), pollinia (SRR5722145), and gynostemium (SRR4431596), were analyzed in this study.

Reads of above-described samples were aligned to the D. catenatum genome [47] using HISAT package [75], which initially remove a portion of the reads based on quality information accompanying each read and then maps the clean reads to the reference genome. StringTie was used to assemble the mapped reads of each sample and estimate the expression levels of all genes by calculating FPKM [76]. The differentially expressed genes were defined with $\mid \log _{2}$ (fold change) $\mid>1$ and with an adjusted $P$-value $(q$-value) $<0.05$ by R package [77]. Heatmap was generated using TBtools software [72].

\section{Quantitative real-time (RT-qPCR) analysis}

Total RNA was extracted using the MiniBEST Plant RNA Extraction Kit (TaKaRa, Japan). The cDNA was reverse transcribed with the PrimerScript RT Enzyme Mix I kit (TaKaRa, Japan). The primers used for expression analysis were designed by Primer Premier 5 (Table S7). qPCR analysis was performed with $\mathrm{SYBR}^{\circledR}$ Premix Ex Taq II (TaKaRa, Japan) on CFX96 Touch $^{\text {TM }}$ Real-Time PCR System (BIO-RAD, USA) in three technical replicates each for three independent biological replicates. The $D c A C$ $T I N$ was used as the internal control gene, which was stably expressed in $D$. catenatum plants and not affected by treatments and genotypes. The relative expression levels were evaluated automatically by the Bio-Rad CFX Manager (version 2.3) with $2^{-\Delta \Delta C \mathrm{~T}}$ method [3].

\footnotetext{
Abbreviations

aa: Amino acid; AMID: Altered MID domain; CDS: Coding sequence; ChIP-Seq: Chromatin immunoprecipitation-based sequencing; CK: Control; CMID: N-terminal cryptic MYC-interaction domain; COI1: CORONATINE INSENSITIVE1; CWDEs: Cell wall-degrading enzymes; DcMTFs: DcMYC2b-targeted TFs; DEGs: Differentiallyexpressed genes; EAR: ETHYLENE-RESPONSE FACTOR Amphiphilic Repression; EGL1: ENHANCER OF GLA BRA3 1; ERF1: Ethylene-ResponseFactor 1; GL3: GLABRA3; HMM: HiddenMarkov model; JAM: JA-ASSOCIATED MYC2-LIKE; Jas: Jasmonates; JAZ: JASMONATE ZIM-DOMAIN proteins; JID: JAZ-interacting domain; LRRs: Leu-richrepeats; MeJA: Methyl jasmonate; MTB MYC2-TARGETED BHLH1; MW: Molecularweights; NINJA: NOVEL INTERACTOR OF JAZ; NJ: Neighbor-joining; ORF: Openreading frame; Pfam: Proteinfamily database; pl: Theoreticalisoelectric points; RT-qPCR: Real-timequantitative polymerase chain reaction; SA: Salicylic acid; SMART: SimpleModular Architecture Research Tool; SRA: SequenceRead Archive; TAD: Transcriptional activation domain; TPL: TOPLESS; TT8: TRANSPARENTTE STA 8; WGD: Whole-genome duplication
}

\section{Supplementary Information}

The online version contains supplementary material available at https://doi. org/10.1186/s12870-021-03134-y.

Additional file 1: Table S1. Characteristics of core JA signaling pathway genes in P. equestris.

Additional file 2: Table S2. Characteristics of core JA signaling pathway genes in A. shenzhenica.

Additional file 3: Figure S1. The leu-rich repeats (LRR) in COI proteins.

Additional file 4: Table S3. Summary of transcriptome sequencing data. Additional file 5: Table S4. Summary of mapped reads in transcriptome sequencing.

Additional file 6: Table S5. Summary of differentially expressed genes in transcriptome analysis.

Additional file 7: Table S6. Expression data of JA signaling pathway genes after MeJA and S. delphinii treatment in D. catenatum.

Additional file 8: Figure $\mathbf{S 2}$. The Mode of JA signaling pathway response to $S$. delphinii in $D$. catenatum.

Additional file 9: Table S7. Primers used for RT-qPCR

\section{Acknowledgements}

We are grateful to Yuqiu Zhu (Zhejiang A\&F University, China) for technical assistance in $D$. catenatum tissue culture process.

\section{Authors' contributions}

$\mathrm{CL}$ and JS designed the experiments and wrote the original draft. QS and $X C$ performed all the experiments. $C L, D L$, and ZH done the formal analysis. LW supplied the pathogen. QS, TZ and DC assisted in editing the article. DC and JS supervised the experiments. All authors have read and approved the manuscript.

\section{Funding}

This research was funded by National Key R\&D Program of China (2017YFC1702201), National Natural Science Foundation of China (31901235), and Scientific R\&D Foundation for Talent Start-up Project of Zhejiang A\&F University (2019FR010). The funding bodies played no role in the design, collection, analysis, interpretation of data, and in writing the manuscript.

\section{Availability of data and materials}

The RNA-seq datasets used this article are available in the NCBI Sequence Read Archive (SRA) with BioProject accession number PRJNA732289. The data that support the conclusions are within this article and its additional files. All data and plant materials used in current study are available from the corresponding author on reasonable request.

\section{Declarations}

\section{Ethics approval and consent to participate}

The materials used in this study is a commercial $D$. catenatum cultivar'Jingpin xianshi', which was cultivated by Prof Jinping Si (Zhejiang A\&F University) and authorized by Zhejiang Province with Breed NO. Zhe S-SV-DC-012-2019. It does not require ethical approval.

\section{Consent for publication}

Not applicable.

\section{Competing interests}

The authors declare that they have no competing interests.

\section{Author details}

${ }^{1}$ State Key Laboratory of Subtropical Silviculture, Zhejiang A\&F University, Hangzhou 311300, China. ${ }^{2}$ College of Agriculture and Biotechnology, Zhejiang University, Hangzhou 310058, China. 
Received: 24 March 2021 Accepted: 25 July 2021

Published online: 06 August 2021

\section{References}

1. Teixeira da Silva JA, Ng TB. The medicinal and pharmaceutical importance of Dendrobium species. Appl Microbiol Biot. 2017;101(6):2227-39.

2. Juswara LS, Schuiteman A, Champion J. Dendrobium annulatum (Orchidaceae. Epidendroideae), a new species of Dendrobium section Grastidium from Indonesian New Guinea. Phytotaxa. 2019:414(3):151-5.

3. Chen DH, Qiu HL, Huang Y, Zhang L, Si JP. Genome-wide identification and expression profiling of SET DOMAIN GROUP family in Dendrobium catenatum. BMC Plant Biol. 2020;20(1):40.

4. Si JP, Wang Q, Liu ZJ, Liu JJ, Luo YB. Breakthrough in key science and technologies in Dendrobium catenatum industry. China J Chin Materia Med. 2017;42(12):2223-7.

5. Chen QY, Chen DH, Shi Y, Si WS, Wu LS, Si JP. Occurrence regularity of Dendrobium catenatum southern blight disease. China J Chin Materia Med. 2019:44(9):1789-92.

6. Iquebal MA, Tomar RS, Parakhia MV, Singla D, Jaiswal S, Rathod VM, Padhiyar SM, Kumar N, Rai A, Kumar D. Draft whole genome sequence of groundnut stem rot fungus Athelia rolfsii revealing genetic architect of its pathogenicity and virulence. Sci Rep. 2017:7(1):5299.

7. LiY, He F, Lai H, Xue Q. Mechanism of in vitro antagonism of phytopathogenic Scelrotium rolfsii by actinomycetes. Eur J Plant Pathol. 2017;149(2):299-311.

8. Jadon KS, Thirumalaisamy PP, Kumar V, Koradia VG, Padavi RD. Management of soil borne diseases of groundnut through seed dressing fungicides. Crop Prot. 2015;78:198-203.

9. Volpiano CG, Lisboa BB, São José JFB, de Oliveira AMR, Beneduzi A, Passaglia LMP, Vargas LK. Rhizobium strains in the biological control of the phytopathogenic fungi Sclerotium (Athelia) rolfsii on the common bean. Plant Soil. 2018;432(1-2):229-43.

10. Hirpara DG, Gajera HP, Hirpara HZ, Golakiya BA. Antipathy of Trichoderma against Sclerotium rolfsii Sacc.: Evaluation of cell wall-degrading enzymatic activities and molecular diversity analysis of antagonists. J Mol Microbiol Biotechnol. 2017;27(1):22-8.

11. Le CN, Kruijt M, Raaijmakers JM. Involvement of phenazines and lipopeptides in interactions between Pseudomonas species and Sclerotium rolfsii, causal agent of stem rot disease on groundnut. J Appl Microbiol. 2012;112(2):390-403.

12. Yan C, Xie D. Jasmonate in plant defence. sentinel or double agent? Plant Biotechnol J. 2015;13(9):1233-40.

13. Bai Y, Meng Y, Huang D, Qi Y, Chen M. Origin and evolutionary analysis of the plant-specific TIFY transcription factor family. Genomics. 2011;98(2):128-36.

14. Guo Q, Major IT, Howe GA. Resolution of growth-defense conflict. mechanistic insights from jasmonate signaling. Curr Opin Plant Biol. 2018:44:72-81.

15. Campos ML, Kang JH, Howe GA. Jasmonate-triggered plant immunity. J Chem Ecol. 2014;40(7):657-75.

16. Fonseca S, Chini A, Hamberg M, Adie B, Porzel A, Kramell R, Miersch O, Wasternack C, Solano R. (+)-7-iso-Jasmonoyl-L-isoleucine is the endogenous bioactive jasmonate. Nat Chem Biol. 2009;5(5):344-50.

17. Devoto A, Nieto-Rostro M, Xie D, Ellis C, Harmston R, Patrick E, Davis J, Sherratt L, Coleman M, Turner JG. COI1 links jasmonate signalling and fertility to the SCF ubiquitin-ligase complex in Arabidopsis. Plant J. 2002;32(4):457-66.

18. Sheard LB, Tan X, Mao H, Withers J, Ben-Nissan G, Hinds TR, Kobayashi Y, Hsu FF, Sharon M, Browse J, et al. Jasmonate perception by inositol-phosphate-potentiated COI1-JAZ co-receptor. Nature. 2010;468(7322):400-5.

19. Thines B, Katsir L, Melotto M, Niu Y, Mandaokar A, Liu G, Nomura K, He SY, Howe GA, Browse J. JAZ repressor proteins are targets of the SCF(COI1) complex during jasmonate signalling. Nature. 2007;448(7154):661-5.

20. Chini A, Fonseca S, Fernandez G, Adie B, Chico JM, Lorenzo O, GarciaCasado G, Lopez-Vidriero I, Lozano FM, Ponce MR, et al. The JAZ family of repressors is the missing link in jasmonate signalling. Nature. 2007;448(7154):666-71.
21. Yan Y, Stolz S, Chetelat A, Reymond P, Pagni M, Dubugnon L, Farmer EE. A downstream mediator in the growth repression limb of the jasmonate pathway. Plant Cell. 2007;19(8):2470-83.

22. Xie DX, Feys BF, James S, Nieto-Rostro M, Turner JG. COl1. An Arabidopsis gene required for jasmonate-regulated defense and fertility. Science. 1998;280(5366):1091-4

23. Vanholme B, Grunewald W, Bateman A, Kohchi T, Gheysen G. The tify family previously known as ZIM. Trends Plant Sci. 2007;12(6):239-44.

24. Melotto M, Mecey C, Niu Y, Chung HS, Katsir L, Yao J, Zeng W, Thines B, Staswick P, Browse J, et al. A critical role of two positively charged amino acids in the Jas motif of Arabidopsis JAZ proteins in mediating coronatine- and jasmonoyl isoleucine-dependent interactions with the COI1 F-box protein. Plant J. 2008;55(6):979-88.

25. Thireault C, Shyu C, Yoshida Y, St Aubin B, Campos ML, Howe GA. Repression of jasmonate signaling by a non-TIFY JAZ protein in Arabidopsis. Plant J. 2015;82(4):669-79.

26. Zhang L, Zhang F, Melotto M, Yao J, He SY. Jasmonate signaling and manipulation by pathogens and insects. J Exp Bot. 2017;68(6):1371-85.

27. Fernandez-Calvo P, Chini A, Fernandez-Barbero G, Chico JM, GimenezIbanez S, Geerinck J, Eeckhout D, Schweizer F, Godoy M, Franco-Zorrilla JM, et al. The Arabidopsis bHLH transcription factors MYC3 and MYC4 are targets of JAZ repressors and act additively with MYC2 in the activation of jasmonate responses. Plant Cell. 2011;23(2):701-15.

28. Bai JF, Wang YK, Guo LP, Guo XM, Guo HY, Yuan SH, Duan WJ, Liu Z, Zhao CP, Zhang FT, et al. Genomic identification and characterization of MYC family genes in wheat (Triticum aestivum L.). BMC Genomics. 2019;20(1):1032.

29. Lorenzo O, Chico JM, Sanchez-Serrano JJ, Solano R. JASMONATE-INSENSITIVE1 encodes a MYC transcription factor essential to discriminate between different jasmonate-regulated defense responses in Arabidopsis. Plant Cell. 2004;16(7):1938-50.

30. Zhang X, Zhu Z, An F, Hao D, Li P, Song J, Yi C, Guo H. Jasmonateactivated MYC2 represses ETHYLENE INSENSITIVE3 activity to antagonize ethylene-promoted apical hook formation in Arabidopsis. Plant Cell. 2014;26(3):1105-17.

31. Du M, Zhao J, Tzeng DTW, Liu Y, Deng L, Yang T, Zhai Q, Wu F, Huang Z, Zhou M, et al. MYC2 Orchestrates a Hierarchical Transcriptional Cascade That Regulates Jasmonate-Mediated Plant Immunity in Tomato. Plant Cell. 2017;29(8):1883-906.

32. Shyu C, Figueroa P, Depew CL, Cooke TF, Sheard LB, Moreno JE, Katsir L, Zheng N, Browse J, Howe GA. JAZ8 lacks a canonical degron and has an EAR motif that mediates transcriptional repression of jasmonate responses in Arabidopsis. Plant Cell. 2012;24(2):536-50.

33. Pauwels L, Barbero GF, Geerinck J, Tilleman S, Grunewald W, Perez AC, Chico JM, Bossche RV, Sewell J, Gil E, et al. NINJA connects the co-repressor TOPLESS to jasmonate signalling. Nature. 2010;464(7289):788-91.

34. Ke JY, Ma HL, Gu X, Thelen A, Brunzelle JS, Li JY, Xu HE, Melcher K. Structural basis for recognition of diverse transcriptional repressors by the TOPLESS family of corepressors. Sci Adv. 2015;1(6):e1500107-e1500107.

35. Chen R, Jiang HL, Li L, Zhai QZ, Qi LL, Zhou WK, Liu XQ, Li HM, Zheng WG, Sun JQ, et al. The Arabidopsis mediator subunit MED25 differentially regulates jasmonate and abscisic acid signaling through interacting with the MYC2 and ABI5 transcription factors. Plant Cell. 2012;24(7):2898-916.

36. Zhang F, Yao J, Ke J, Zhang L, Lam VQ, Xin XF, Zhou XE, Chen J, Brunzelle J, Griffin PR, et al. Structural basis of JAZ repression of MYC transcription factors in jasmonate signalling. Nature. 2015;525(7568):269-73.

37. Liu Y, Du M, Deng L, Shen J, Fang M, Chen Q, Lu Y, Wang Q, Li C, Zhai Q. MYC2 regulates the termination of jasmonate signaling via an autoregulatory negative feedback loop. Plant Cell. 2019;31(1):106-27.

38. Chung HS, Howe GA. A critical role for the TIFY motif in repression of jasmonate signaling by a stabilized splice variant of the JASMONATE ZIMdomain protein JAZ10 in Arabidopsis. Plant Cell. 2009;21(1):131-45.

39. Chung HS, Cooke TF, Depew CL, Patel LC, Ogawa N, Kobayashi Y, Howe GA. Alternative splicing expands the repertoire of dominant JAZ repressors of jasmonate signaling. Plant J. 2010;63(4):613-22.

40. Moreno JE, Shyu C, Campos ML, Patel LC, Chung HS, Yao J, He SY, Howe GA. Negative feedback control of jasmonate signaling by an alternative splice variant of JAZ10. Plant Physiol. 2013;162(2):1006-17.

41. Goossens J, Swinnen G, Vanden Bossche R, Pauwels L, Goossens A. Change of a conserved amino acid in the MYC2 and MYC3 transcription factors leads to release of JAZ repression and increased activity. New Phytol. 2015;206(4):1229-37. 
42. Howe GA, Major IT, Koo AJ. Modularity in Jasmonate Signaling for Multistress Resilience. Annu Rev Plant Biol. 2018;69:387-415.

43. Yan JB, Zhang C, Gu M, Bai ZY, Zhang WG, Qi TC, Cheng ZW, Peng W, Luo $\mathrm{HB}$, Nan FJ, et al. The Arabidopsis CORONATINE INSENSITIVE1 protein is a jasmonate receptor. Plant Cell. 2009;21 (8):2220-36.

44. Song SS, Qi TC, Wasternack C, Xie DX. Jasmonate signaling and crosstalk with gibberellin and ethylene. Curr Opin Plant Biol. 2014;21:112-9.

45. Kazan K, Manners JM. MYC2: the master in action. Mol Plant. 2013:6(3):686-703.

46. Zhang GQ, Liu KW, Li Z, Lohaus R, Hsiao YY, Niu SC, Wang JY, Lin YC, Xu $\mathrm{Q}$, Chen $\mathrm{L}$, et al. The Apostasia genome and the evolution of orchids. Nature. 2017;549(7672):379-83.

47. Zhang GQ, Xu Q, Bian C, Tsai WC, Yeh CM, Liu KW, Yoshida K, Zhang LS, Chang SB, Chen F, et al. The Dendrobium catenatum Lindl. genome sequence provides insights into polysaccharide synthase, floral development and adaptive evolution. Sci Rep. 2016;6:19029.

48. Cai J, Liu X, Vanneste K, Proost S, Tsai WC, Liu KW, Chen LJ, He Y, Xu Q, Bian $C$, et al. The genome sequence of the orchid Phalaenopsis equestris. Nat Genet. 2015;47(1):65-72.

49. Xia W, Yu H, Cao P, Luo J, Wang N. Identification of TIFY family genes and analysis of their expression profiles in response to phytohormone treatments and melampsora larici-populina infection in Poplar. Front Plant Sci. 2017;8:493.

50. Liu X, Zhao C, Yang L, Zhang Y, Wang Y, Fang Z, Lv H. Genome-Wide identification, expression profile of the TIFY gene family in Brassica oleracea var. capitata, and their divergent response to various pathogen infections and phytohormone treatments. Genes (Basel). 2020;11(2):127.

51. Ye H, Du H, Tang N, Li X, Xiong L. Identification and expression profiling analysis of TIFY family genes involved in stress and phytohormone responses in rice. Plant Mol Biol. 2009;71(3):291-305.

52. Chini A, Ben-Romdhane W, Hassairi A, Aboul-Soud MAM. Identification of TIFY/JAZ family genes in Solanum lycopersicum and their regulation in response to abiotic stresses. PLoS One. 2017;12(6):e0177381.

53. Feys B, Benedetti CE, Penfold CN, Turner JG. Arabidopsis mutants selected for resistance to the phytotoxin coronatine are male sterile, insensitive to methyl jasmonate, and resistant to a bacterial pathogen. Plant Cell. 1994;6(5):751-9.

54. Xu L, Liu F, Lechner E, Genschik P, Crosby WL, Ma H, Peng W, Huang D, Xie D. The SCF (COI1) ubiquitin-ligase complexes are required for jasmonate response in Arabidopsis. Plant Cell. 2002;14(8):1919-35.

55. Lee HY, Seo JS, Cho JH, Jung H, Kim JK, Lee JS, Rhee S, Do Choi Y. Oryza sativa COI homologues restore jasmonate signal transduction in Arabidopsis coil-1 mutants. PLoS One. 2013;8(1):e52802.

56. Yang DL, Yao J, Mei CS, Tong XH, Zeng LJ, Li Q, Xiao LT, Sun TP, Li J, Deng $\mathrm{XW}$, et al. Plant hormone jasmonate prioritizes defense over growth by interfering with gibberellin signaling cascade. Proc Natl Acad Sci U S A. 2012;109(19):E1192-1200

57. Ye M, Luo SM, Xie JF, Li YF, XuT, Liu Y, Song YY, Zhu-Salzman K, Zeng RS. Silencing COl1 in rice increases susceptibility to chewing insects and impairs inducible defense. PLoS One. 2012;7(4):e36214.

58. Clark JW, Donoghue PCJ. Whole-genome duplication and plant macroevolution. Trends Plant Sci. 2018;23(10):933-45.

59. Lynch M, Conery JS. The evolutionary fate and consequences of duplicate genes. Science. 2000;290(5494):1151-5.

60. Feng S, Ma L, Wang X, Xie D, Dinesh-Kumar SP, Wei N, Deng XW. The COP9 signalosome interacts physically with SCF COI1 and modulates jasmonate responses. Plant Cell. 2003;15(5):1083-94.

61. Hou X, Lee LY, Xia K, Yan Y, Yu H. DELLAs modulate jasmonate signaling via competitive binding to JAZs. Dev Cell. 2010;19(6):884-94.
62. Jiang Y, Liang G, Yang S, Yu D. Arabidopsis WRKY57 functions as a node of convergence for jasmonic acid- and auxin-mediated signaling in jasmonic acid-induced leaf senescence. Plant Cell. 2014;26(1):230-45.

63. Wasternack C, Song S. Jasmonates: biosynthesis, metabolism, and signaling by proteins activating and repressing transcription. J Exp Bot. 2017;68(6):1303-21.

64. Dombrecht B, Xue GP, Spraque SJ, Kirkegaard JA, Ross JJ, Reid JB, Fitt GP, Sewelam N, Schenk PM, Manners JM, et al. MYC2 differentially modulates diverse jasmonate-dependent functions in Arabidopsis. Plant Cell. 2007;19(7):2225-45

65. Nakata M, Mitsuda N, Herde M, Koo AJ, Moreno JE, Suzuki K, Howe GA, Ohme-Takagi M. A bHLH-type transcription factor, ABA-INDUCIBLE BHLHTYPE TRANSCRIPTION FACTOR/JA-ASSOCIATED MYC2-LIKE1, acts as a repressor to negatively regulate jasmonate signaling in Arabidopsis. Plant Cell. 2013;25(5):1641-56.

66. Qi T, Wang J, Huang H, Liu B, Gao H, Liu Y, Song S, Xie D. Regulation of jasmonate-induced leaf senescence by antagonism between bHLH subgroup Ille and IIId factors in Arabidopsis. Plant Cell. 2015;27(6):1634-49.

67. Wu LS, Dong WG, Si JP, Liu JJ, Zhu YQ. Endophytic fungi, host genotype, and their interaction influence the growth and production of key chemical components of Dendrobium catenatum. Fungal Biol. 2020;124(10):864-76.

68. Berrocal-Lobo M, Molina A, Solano R. Constitutive expression of ETHYLENE-RESPONSE-FACTOR1 in Arabidopsis confers resistance to several necrotrophic fungi. Plant J. 2002;29(1):23-32.

69. Lee SH, Sakuraba Y, Lee T, Kim KW, An G, Lee HY, Paek NC. Mutation of Oryza sativa CORONATINE INSENSITIVE $1 \mathrm{~b}$ (OsCOI1 b) delays leaf senescence. J Integr Plant Biol. 2015;57(6):562-76.

70. Finn RD, Coggill P, Eberhardt RY, Eddy SR, Mistry J, Mitchell AL, Potter SC, Punta M, Qureshi M, Sangrador-Vegas A, et al. The Pfam protein families database. towards a more sustainable future. Nucleic Acids Res. 2016;44(D1):D279-285.

71. Finn RD, Clements J, Eddy SR. HMMER web server: interactive sequence similarity searching. Nucleic Acids Res. 2011;39(Web Server issue):W29-37.

72. Chen CJ, Chen H, Zhang Y, Thomas HR, Frank MH, He YH, Xia R. TBtools. An integrative toolkit developed for interactive analyses of big biological data. Mol Plant. 2020;13(8):1 194-202.

73. Larkin MA, Blackshields G, Brown NP, Chenna R, McGettigan PA, McWilliam H, Valentin F, Wallace IM, Wilm A, Lopez R, et al. Clustal W and clustal $X$ version 2.0. Bioinformatics. 2007;23(21):2947-8.

74. Kumar S, Stecher G, Tamura K. MEGA7. Molecular evolutionary genetics analysis version 7.0 for bigger datasets. Mol Biol Evol. 2016;33(7):1870-4.

75. Kim D, Landmead B, Salzberg SL. HISAT: a fast spliced aligner with low memory requirements. Nat Methods. 2015;12(4):357-U121.

76. Pertea M, Pertea GM, Antonescu CM, Chang TC, Mendell JT, Salzberg SL. StringTie enables improved reconstruction of a transcriptome from RNAseq reads. Nat Biotechnol. 2015;33(3):290.

77. Anders S, Huber W. Differential expression analysis for sequence count data. Genome Biol. 2010;11(10):R106.

\section{Publisher's Note}

Springer Nature remains neutral with regard to jurisdictional claims in published maps and institutional affiliations.

Ready to submit your research? Choose BMC and benefit from:

- fast, convenient online submission

- thorough peer review by experienced researchers in your field

- rapid publication on acceptance

- support for research data, including large and complex data types

- gold Open Access which fosters wider collaboration and increased citations

- maximum visibility for your research: over 100M website views per year

At BMC, research is always in progress.

Learn more biomedcentral.com/submissions 